\title{
Evaluación de la eficiencia de tres modelos de incubadora con diferentes flujos en el desarrollo larval de Osteoglossum bicirrhosum (arawana plateada)
}

\author{
EVALUATION OF THE EFFICIENCY OF THREE INCUBATOR MODELS WITH DIFFERENT FLOWS IN \\ LARVAL DEVELOPMENT OF Osteoglossum bicirrhosum (SILVER AROWANA)
}

Sergio Luis Oliveros ${ }^{1}$, Kelly Johana Hernández Paniagua ${ }^{1}$, Gustavo Adolfo PimentelParra $^{2,3}$, Betselene Murcia-Ordoñez ${ }^{2}$, Luis Carlos Chaves-Moreno ${ }^{1}$

\section{RESUMEN}

El objetivo de este estudio fue evaluar la eficiencia de tres modelos de incubadora con diferente flujo para el desarrollo larval de Osteoglossum bicirrhosum (arawana plateada), desde el estadio dos al estadio cinco. Se trabajó con 60 larvas y se distribuyeron en grupos de 20 por cada modelo de incubadora: MI1: flujo circular; MI2: flujo ascendente; MI3: sin flujo. Se determinó el diámetro corporal (DC), diámetro del saco vitelino (DSV), adquisición natatoria (AN) y mortalidad durante 30 días de incubación. El DC final para MI1 fue de 33.77 mm comparado con 33.16 mm para MI2 y $19.92 \mathrm{~mm}$ para MI3. El DSV inicial de $12.02 \mathrm{~mm}$ en MI1 disminuyó en MI2 (11.64 mm) y aumentó a $12.11 \mathrm{~mm}$ en MI3. La adquisición natatoria se presentó en todas las larvas de MI1 y MI2, pero solo en el 10\% de MI3. Asimismo, la mortalidad fue de 1, 10 y 20 larvas para MI1, MI2 y MI3, respectivamente.

Palabras clave: incubadora; diámetro corporal; diámetro del saco vitelino; adquisición natatoria; mortalidad

\section{ABstract}

The aim of this study was to evaluate the efficiency of three incubator models with different flow for the larval development of Osteoglossum bicirrhosum (silver arowana) from stage two to stage five. Sixty larvae were distributed in groups of 20 for each model

\footnotetext{
${ }^{1}$ Grupo de Investigación BYDA, Programa de Medicina Veterinaria y Zootecnia, Facultad de Ciencias Agropecuarias, Universidad de la Amazonia, Florencia, Colombia

${ }^{2}$ Grupo de Investigación BYDA, Programa de Biología, Facultad de Ciencias Básicas, Universidad de la Amazonia, Florencia, Colombia

${ }^{3}$ E-mail: gustavoadolfopimentelparra@hotmail.com
}

Recibido: 25 de noviembre de 2016

Aceptado para publicación: 15 de diciembre de 2017 
of incubator: MI1: circular flow; MI2: upflow; MI3: no flow). Body diameter (DC), yolk sac diameter (DSV), swimming acquisition (AN) and mortality during 30 days of incubation were determined. The final DC for MI 1 was $33.77 \mathrm{~mm}$ compared to $33.16 \mathrm{~mm}$ for MI2 and $19.92 \mathrm{~mm}$ for MI3. The initial DSV in MI1 of $12.03 \mathrm{~mm}$ decreased to $11.64 \mathrm{~mm}$ in MI2 and increased to $12.11 \mathrm{~mm}$ in MI3. The AN occurred in all larvae of MI1 and MI2, but only in $10 \%$ of MI3. Likewise, mortality was 1, 10 and 20 larvae for MI1, MI2 and MI3, respectively.

Key words: incubator; body diameter; yolk sac diameter; swimming acquisition; mortality

\section{INTRODUCCIÓN}

La producción piscícola en Colombia ha adquirido un gran potencial como fuente alternativa de alimentos de origen animal. Debido a esto, especies ícticas como arawana plateada (Osteoglossum bicirrhosum) y pirarucú o paiche (Arapaima sp) se han convertido en fuentes alternas de alimento que se pueden cultivar en áreas no aptas o de bajo rendimiento para otras actividades agrícolas y pecuarias (suelos pobres en nutrientes) (Quintero-Pinto et al., 2011).

Uno de los procesos limitantes en la piscicultura es la reproducción e incubación de los individuos (Salinas-Coy y AgudeloCórdoba, 2000; Ribeyro-Schult et al., 2014). Las condiciones requeridas para la incubación incluyen factores como la temperatura, la intensidad lumínica, la calidad del agua, el tipo de sustrato y el nivel de estrés a que son sometidos los huevos (Carillo-Estévez, 2009; Hernández-Olaya et al., 2010).

En peces como la arawana, la incubación natural ocurre en la cavidad bucal de los machos, la cual tiene la capacidad de albergar a los alevinos hasta que son capaces de valerse por sí mismos (Bonilla-Castillo y Agudelo, 2012; Garzón-Ortíz et al., 2015). El proceso de incubación natural dura alrededor de 45 días, donde los machos no consumen alimento (Landines-Parra et al., 2007; Hernández-Olaya, 2009). Cuando las larvas son extraídas de sus parentales en un estadio más temprano, requieren de incuba-ción artificial, teniendo que adecuarse las condicio- nes del microhábitat a una temperatura, flujograma y niveles de oxígeno adecuados que permitan obtener el mayor porcentaje de supervivencia (Prieto y Olivera, 2002; PieroGhezzi et al., 2015).

Algunos trabajos que se han desarrollado en incubación artificial tienen el mismo principio de flujo ascendente, los cuales se realizan con incubadoras tipo embudo o Woynarovich (Monaco y Doroshv, 1983; Argumedo y Rojas, 2000; Balbuena et al., 2011; Chaves et al., 2011).

El objetivo del estudio fue evaluar la eficiencia de tres modelos de incubadora con diferente flujo de agua con respecto al diámetro corporal (DC), diámetro del saco vitelino (DSV), adquisición natatoria (AN) y mortalidad para el desarrollo larval de $O$. bicirrhosum.

\section{MATERIAles y MéTOdos}

La investigación se desarrolló en el laboratorio de Investigación de Ictiología (LIUA), ubicado en la sede centro de la Universidad de la Amazonia de la ciudad de Florencia, Caquetá (Colombia). La zona tiene una altitud promedio de $377 \mathrm{~m}$, humedad relativa de $80-85 \%$, temperatura ambiente promedio de $28^{\circ} \mathrm{C}$, precipitación anual de $3835 \mathrm{~mm}$ y brillo solar aproximado de 2000 horas/año. Para el desarrollo de la investigación se contó con el permiso de bioética otorgado por la Facultad de Ciencias Agropecuarias de la Universidad de la Amazonia. 
Las larvas se obtuvieron en una producción piscícola ubicada en el municipio de San José del Fragua, Caquetá, que cuenta con 30 reproductores de $O$. bicirrhosum de aproximadamente siete años. La captura se hizo por medio de la utilización de chinchorro y la participación de cuatro personas. Se capturaron tres machos, se los cuales se seleccionaron 60 larvas de la cavidad bucal por macho (las cuales son recolectadas de sus parentales abriendo la boca de los machos para liberar los alevinos sin necesidad de un anestésico). Las larvas se encontraban en estadio dos de alevinaje (presencia de desarrollo de cerebro y cráneo desarrollado, ojos desarrollados, se observan arcos y lamelas branquiales y saco vitelino vascularizado) (Argumedo-Trilleras et al., 2007, 2017; Argumedo-Trilleras, 2009). Las larvas se depositaron en una bolsa plástica trasparente de fondo cuadrado de $10 \mathrm{~L}$ ( $1 / 4$ de agua y $3 / 4$ de oxígeno) para su transporte al laboratorio LI-UA (Piero-Ghezzi et al., 2015; MuñozTrujillo et al., 2017).

En el laboratorio, las larvas se depositaron en un sistema cerrado de recirculación (SCR), que cuenta con temperatura constante de $27^{\circ} \mathrm{C}$ y pH 6.5 (Argumedo-Trilleras, 2009; Argumedo-Trilleras et al., 2017). La población se distribuyó en tres modelos de incubadoras (MI) con la misma estructura (esférica), capacidad (1.5 L) y entrada de agua $(0.012 \mathrm{~L} / \mathrm{s})$. En cada MI se colocaron 20 larvas (Figura 1).

El MI1 está constituido por un sistema de flujo circular permitiendo que las larvas se muevan de forma suave, en círculos y en forma constante, simulando la incubación bucal del macho en su habitad natural (Hernández-Olaya, 2009). El MI2 presenta un modelo de flujo ascendente, causado por una piedra oxigenadora ubicada en la parte media y superficial de la esfera, donde el flujo permite que las larvas se muevan de manera ascendente y continua en el mismo punto, pero sin rotación. El MI3 es un modelo simple que no cuenta con algún tipo de flujo, de manera que las larvas permanecen sin movimiento.
La investigación tuvo una duración de 30 días. Durante este periodo se extrajo diariamente, con un ansa de 8 pulgadas, a cinco individuos al azar de cada MI para medir, con un pie de rey o calibrador digital, el diámetro corporal (DC) que corresponde desde el inicio de la boca hasta la terminación de la aleta caudal y el diámetro del saco vitelino (DSV) que corresponde a la unión del sistema digestivo hasta la terminación de la zona caudal del saco. También se monitoreó la adquisición natatoria y la mortalidad.

El análisis estadístico se efectuó por medio de un análisis de varianza con prueba de separación de medias LSD de Fisher, con un nivel de confiabilidad del $95 \%$ para todas las variables evaluadas por incubadora y por el tiempo (días). De igual forma, se efectuó un análisis de componentes principales (ACP) para la incubadora y para el tiempo (días). Todos los análisis fueron hechos en el programa InfoStat versión profesional 2017.

\section{Resultados y Discusión}

Se encontró diferencia significativa en diámetro corporal por el tipo de incubadora $(p<0.05)$, presentándose el mayor diámetro en MI1, flujo circular (33.77 \pm 0.14$)$ y el menor diámetro en MI3, sin flujo (19.92 \pm 0.22 ). Con relación al tiempo (días) se presentó diferencias significativas $(p<0.05)$, donde el valor más alto se dio en el día $30(55.35 \pm$ $0.54 \mathrm{~mm}$ en y el menor valor el día 2 (13.68 \pm $0.44 \mathrm{~mm}$ ) (Cuadro 1). El DC obtenido en los tres tipos de incubadora para el estadio larval cinco corresponden al tiempo promedio normal de una incubación natural, lo cual indica que el tipo de flujo no afectó la duración del desarrollo larval de O. bicirrhosum (Argumedo-Trilleras et al., 2007; ArgumedoTrilleras, 2009).

La variable diámetro del saco vitelino (DSV) mostró diferencias significativas $(\mathrm{p}<0.05)$, donde el valor más alto se evidenció en MI3, sin flujo $(12.11 \pm 0.12 \mathrm{~mm})$ y el menor valor en MI2, flujo oxigenado (11.64 \pm 


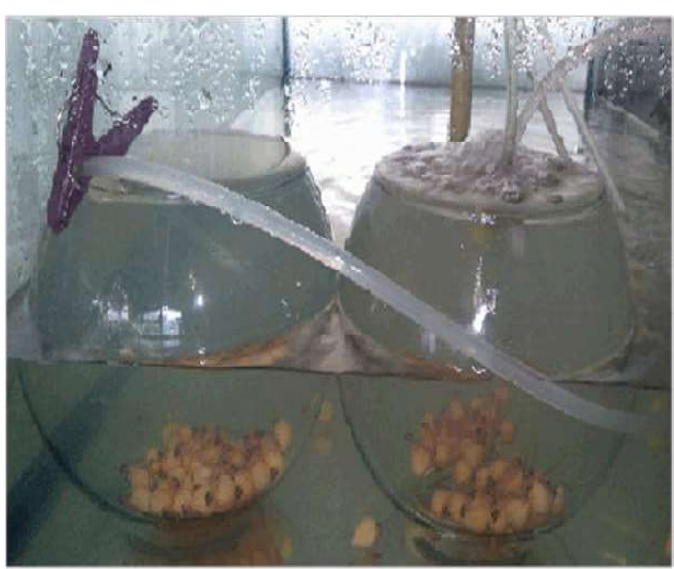

Figura 1. Sistema de recirculación cerrada (SRC) utilizados en las incubadoras para larvas de Osteoglossum bicirrhosum

$0.08 \mathrm{~mm}$ ), pero sin presentar diferencias significativas por los días de incubación (Cuadro 1). Se debe resaltar la forma redondeada que tomaron los sacos vitelinos de los tres grupos, posiblemente debido a que las larvas se desarrollaron en un espacio mayor al que lo harían en la incubación bucal, donde la forma del saco vitelino es alargada. El macho puede albergar entre 60 a 220 larvas en la cavidad bucal, e incluso más (ArgumedoTrilleras, 2009; Garzón-Ortíz et al., 2015), causando que estén en un ambiente más estrecho que el proporcionado en las incubadoras.

La mayor mortalidad se presentó en MI3, sin flujo (2.46 $\pm 0.30 \%)$ y la menor en MI1, flujo circular $(0.07 \pm 0.19 \%)$ y MI2, flujo oxigenado $(0.54 \pm 0.19)(\mathrm{p}<0.05)$. Asimismo, el mayor valor de mortalidad se evidenció en el día $4(4.50 \pm 0.60)$ y el menor valor en el día 30 de incubación $(0.00001 \pm 0.73)$ (Cuadro 1).

La adquisición natatoria también mostró diferencias estadísticas significativas $(\mathrm{p}<0.05)$, tanto para incubadora como para el tiempo. La incubadora con los valores más altos fue MI1, flujo circular $(2.35 \pm 0.31)$ y el menor valor en MI3, sin flujo (0.0001 \pm 0.48$)$. Asimismo, la mayor adquisición natatoria se evidenció en el día $30(27.00 \pm 1.19)$ y el menor valor en el día $14(0.22 \pm 1.37)$ (Cuadro 1).

El análisis de componentes principales (ACP) efectuado sobre el desarrollo de los alevinos incubados en tres modelos de incubadora mostró que los dos primeros componentes principales (CP) aportan el 100\% de la variabilidad total. Al analizar los coeficientes de los vectores asociados a las dos primeros componentes principales se encontró que las variables de mayor importancia fueron la mortalidad y el diámetro corporal. Según la Figura 2, el primer componente principal (CP1), con un aporte del $75.2 \%$ separó a la incubadora MI3 (sin flujo) asociada al extremo positivo de dicho componente con las variables mortalidad y $\mathrm{pH}$ del MI1 (flujo circular) asociada al extremo negativo de dicho componente con las variables adquisición natatoria y diámetro corporal. Así mismo, el segundo componente principal (CP2) con un aporte del $24.8 \%$ agrupa a la incubadora MI2 (flujo oxigenado) que se asocia al extremo negativo de dicho componente con la variable temperatura; mientras que la variable diámetro del saco vitelino no se asocia con ninguna de las incubadoras.

El análisis de componentes principales (ACP) con respecto al tiempo de incubación (días), mostró que los tres primeros componentes principales (CP) aportan el $80 \%$ de la variabilidad total. Al analizar los coeficientes de los vectores asociados a los dos primeros componentes principales se encontró que las variables de mayor importancia fueron el diámetro corporal y la adquisición natatoria. Según la Figura 3, el primer componente principal (CP1) con un aporte del $41.7 \%$ al extremo positivo de dicho componente se asocia con las variables adquisición natatoria con los días 26, 29 y 30 donde se presentó los mayores picos. En cuanto a la variable diámetro corporal (DC), los mayores valores se presentaron en los días 25, 27 y 28 . Asimismo, al extremo negativo de dicho componente, la 
Cuadro 1. Diámetro corporal (DC), diámetro del saco vitelino (DSV), mortalidad, adquisición natatoria (A-natatoria) (media \pm error estándar) de alevinos de Osteoglossum bicirrhosum del estadio larval dos al cinco, incubados por 30 días en tres modelos de incubadora (MI1: flujo circular; MI2: flujo oxigenado; MI3: sin flujo)

\begin{tabular}{|c|c|c|c|c|c|}
\hline \multirow[t]{2}{*}{ Factor } & \multirow{2}{*}{ Nivel } & \multicolumn{4}{|c|}{ Variables $^{1}$} \\
\hline & & $\mathrm{DC}(\mathrm{mm})$ & DSV (mm) & Mortalidad & A-Natatoria \\
\hline \multirow[t]{3}{*}{ Incubadora } & MI1 & $33.77 \pm 0.14^{c}$ & $12.02 \pm 0.07^{b}$ & $0.07 \pm 0.19^{\mathrm{a}}$ & $2.35 \pm 0.31^{b}$ \\
\hline & MI2 & $33.16 \pm 0.14^{\mathrm{b}}$ & $11.64 \pm 0.08^{a}$ & $0.54 \pm 0.19^{\mathrm{a}}$ & $1.05 \pm 0.31^{\mathrm{a}}$ \\
\hline & MI3 & $19.92 \pm 0.22^{\mathrm{a}}$ & $12.11 \pm 0.12^{\mathrm{b}}$ & $2.46 \pm 0.30^{\mathrm{b}}$ & $0.00 \pm 0.48^{\mathrm{a}}$ \\
\hline \multirow{30}{*}{$\begin{array}{l}\text { Días de } \\
\text { incubación }\end{array}$} & 1 & $14.03 \pm 0.44^{\mathrm{a}}$ & $12.14 \pm 0.24$ & $12.14 \pm 0.24^{\mathrm{be}}$ & $0.00 \pm 0.97^{\mathrm{a}}$ \\
\hline & 2 & $13.68 \pm 0.44^{\mathrm{a}}$ & $11.95 \pm 0.24$ & $11.95 \pm 0.24^{\mathrm{a}-\mathrm{e}}$ & $0.00 \pm 0.97^{\mathrm{a}}$ \\
\hline & 3 & $16.18 \pm 0.44^{\mathrm{b}}$ & $12.32 \pm 0.24$ & $12.32 \pm 0.24^{\mathrm{de}}$ & $0.00 \pm 0.97 \mathrm{a}$ \\
\hline & 4 & $18.35 \pm 0.44^{\mathrm{c}}$ & $12.52 \pm 0.24$ & $12.52 \pm 0.24^{\mathrm{e}}$ & $0.00 \pm 0.97^{\mathrm{a}}$ \\
\hline & 5 & $19.02 \pm 0.44^{\mathrm{c}}$ & $11.87 \pm 0.24$ & $11.87 \pm 0.24^{\mathrm{a}-\mathrm{e}}$ & $0.00 \pm 0.97^{\mathrm{a}}$ \\
\hline & 6 & $20.33 \pm 0.44^{\mathrm{d}}$ & $11.92 \pm 0.24$ & $11.92 \pm 0.24^{\mathrm{a}-\mathrm{e}}$ & $0.00 \pm 0.97^{a}$ \\
\hline & 7 & $21.58 \pm 0.44^{\mathrm{e}}$ & $11.93 \pm 0.24$ & $11.93 \pm 0.24^{\mathrm{a}-\mathrm{e}}$ & $0.00 \pm 0.97^{\mathrm{a}}$ \\
\hline & 8 & $22.48 \pm 0.44^{\mathrm{e}}$ & $11.92 \pm 0.24$ & $11.92 \pm 0.24^{\mathrm{a}-\mathrm{e}}$ & $0.00 \pm 0.97^{\mathrm{a}}$ \\
\hline & 9 & $24.58 \pm 0.44 f$ & $11.57 \pm 0.24$ & $11.57 \pm 0.24^{\mathrm{a}-\mathrm{c}}$ & $0.00 \pm 0.97^{\mathrm{a}}$ \\
\hline & 10 & $25.95 \pm 0.44^{\mathrm{g}}$ & $11.55 \pm 0.24$ & $11.55 \pm 0.24^{\mathrm{a}-\mathrm{c}}$ & $0.00 \pm 0.97^{\mathrm{a}}$ \\
\hline & 11 & $26.58 \pm 0.44^{\mathrm{gh}}$ & $11.82 \pm 0.24$ & $11.82 \pm 0.24^{\mathrm{a}-\mathrm{e}}$ & $0.00 \pm 0.97^{a}$ \\
\hline & 12 & $27.68 \pm 0.44^{\mathrm{h}}$ & $11.73 \pm 0.24$ & $11.73 \pm 0.24^{\mathrm{a}-\mathrm{d}}$ & $0.00 \pm 0.97^{\mathrm{a}}$ \\
\hline & 13 & $29.93 \pm 0.54^{\mathrm{i}}$ & $11.83 \pm 0.29$ & $11.83 \pm 0.29^{\mathrm{a}-\mathrm{e}}$ & $0.00 \pm 1.19^{\mathrm{a}}$ \\
\hline & 14 & $30.26 \pm 0.63^{\mathrm{i}}$ & $11.97 \pm 0.33$ & $11.97 \pm 0.33^{\mathrm{a}-\mathrm{e}}$ & $0.22 \pm 1.37^{\mathrm{a}}$ \\
\hline & 15 & $32.35 \pm 0.54^{j}$ & $12.03 \pm 0.29$ & $12.03 \pm 0.29^{\mathrm{b}-\mathrm{e}}$ & $0.00 \pm 1.19^{\mathrm{a}}$ \\
\hline & 16 & $34.35 \pm 0.54^{\mathrm{k}}$ & $11.80 \pm 0.29$ & $11.80 \pm 0.29^{\mathrm{a}-\mathrm{e}}$ & $0.00 \pm 1.19^{\mathrm{a}}$ \\
\hline & 17 & $35.40 \pm 0.54^{\mathrm{kl}}$ & $11.93 \pm 0.29$ & $11.93 \pm 0.29^{\mathrm{a}-\mathrm{e}}$ & $1.00 \pm 1.19^{\mathrm{a}}$ \\
\hline & 18 & $36.53 \pm 0.54^{\mathrm{lm}}$ & $11.83 \pm 0.29$ & $11.83 \pm 0.29^{\mathrm{a}-\mathrm{e}}$ & $0.00 \pm 1.19^{\mathrm{a}}$ \\
\hline & 19 & $37.80 \pm 0.54^{\mathrm{m}}$ & $12.15 \pm 0.29$ & $12.15 \pm 0.29^{\mathrm{b}-\mathrm{e}}$ & $0.00 \pm 1.19^{\mathrm{a}}$ \\
\hline & 20 & $39.50 \pm 0.54^{\mathrm{n}}$ & $11.75 \pm 0.29$ & $11.75 \pm 0.29^{\mathrm{a}-\mathrm{d}}$ & $0.00 \pm 1.19 \mathrm{a}$ \\
\hline & 21 & $41.10 \pm 0.54^{\circ}$ & $11.68 \pm 0.29$ & $11.68 \pm 0.29^{\mathrm{a}-\mathrm{d}}$ & $0.00 \pm 1.19^{\mathrm{a}}$ \\
\hline & 22 & $42.23 \pm 0.54^{\circ}$ & $11.90 \pm 0.29$ & $11.90 \pm 0.29^{\mathrm{a}-\mathrm{e}}$ & $0.00 \pm 1.19^{\mathrm{a}}$ \\
\hline & 23 & $43.75 \pm 0.54^{\mathrm{p}}$ & $12.25 \pm 0.29$ & $12.25 \pm 0.29^{\mathrm{c}-\mathrm{e}}$ & $0.00 \pm 1.19^{\mathrm{a}}$ \\
\hline & 24 & $44.75 \pm 0.54^{\mathrm{pq}}$ & $12.13 \pm 0.29$ & $12.13 \pm 0.29^{\mathrm{b}-\mathrm{e}}$ & $0.00 \pm 1.19^{\mathrm{a}}$ \\
\hline & 25 & $45.93 \pm 0.54^{\mathrm{qr}}$ & $11.83 \pm 0.29$ & $11.83 \pm 0.29^{\mathrm{a}-\mathrm{e}}$ & $0.00 \pm 1.19^{\mathrm{a}}$ \\
\hline & 26 & $46.75 \pm 0.54^{\mathrm{r}}$ & $11.88 \pm 0.29$ & $11.88 \pm 0.29^{\mathrm{a}-\mathrm{e}}$ & $11.25 \pm 1.19^{b}$ \\
\hline & 27 & $49.58 \pm 0.54^{\mathrm{s}}$ & $11.80 \pm 0.29$ & $11.80 \pm 0.29^{\mathrm{a}-\mathrm{e}}$ & $0.00 \pm 1.19^{\mathrm{a}}$ \\
\hline & 28 & $50.75 \pm 0.54^{\mathrm{st}}$ & $11.40 \pm 0.29$ & $11.40 \pm 0.29^{\mathrm{ab}}$ & $0.00 \pm 1.19^{\mathrm{a}}$ \\
\hline & 29 & $51.35 \pm 0.54^{\mathrm{t}}$ & $11.43 \pm 0.29$ & $11.43 \pm 0.29^{\mathrm{ab}}$ & $12.00 \pm 1.19^{b}$ \\
\hline & 30 & $55.35 \pm 0.54^{\mathrm{u}}$ & $11.20 \pm 0.29$ & $11.20 \pm 0.29^{\mathrm{a}}$ & $27.00 \pm 1.19^{c}$ \\
\hline
\end{tabular}

Valores con letras diferentes dentro de columnas son estadísticamente diferentes $(p<0.05)$ 


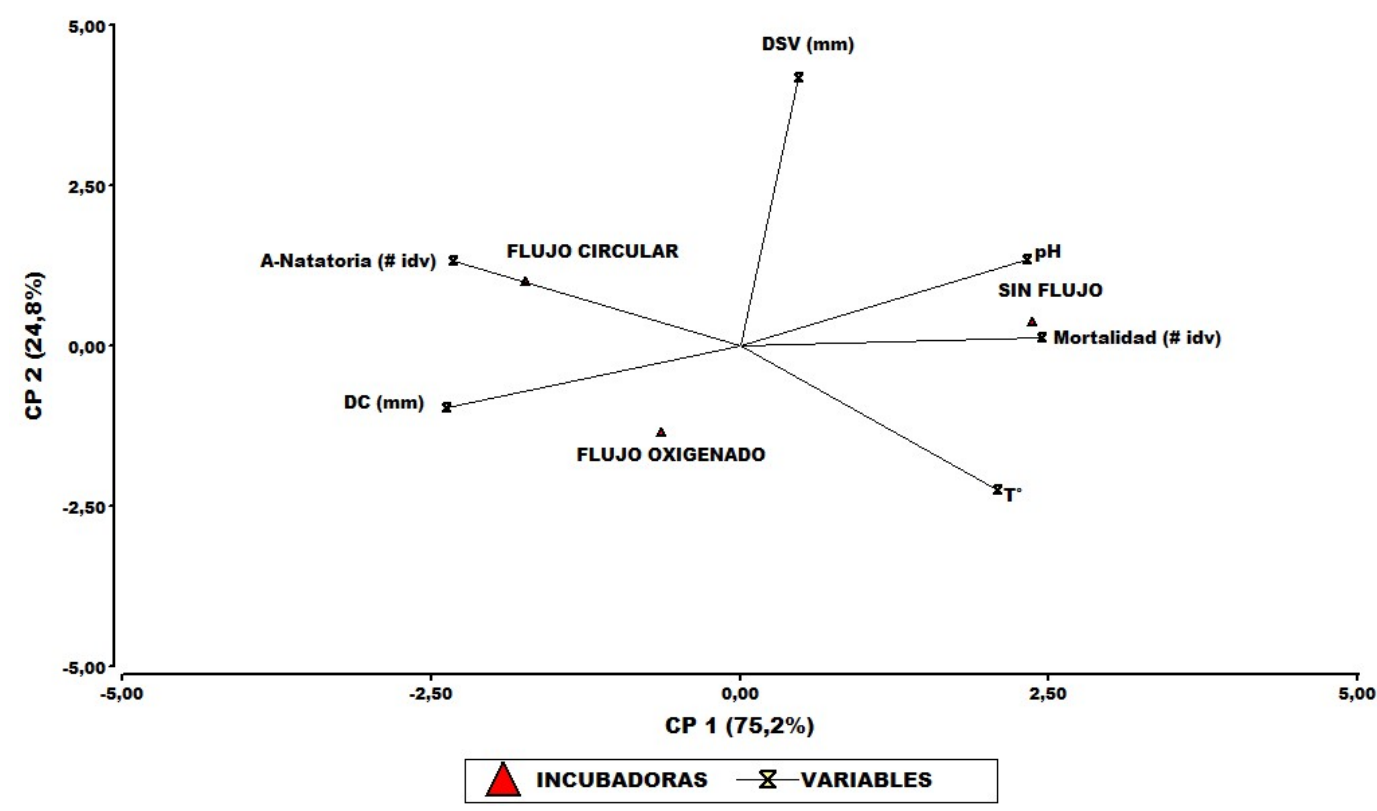

Figura 2. Análisis de componentes principales (ACP) con grafico biplot de alevinos de Osteoglossum bicirrhosum del estadio larval dos al cinco, incubados en tres modelos de incubadora (MI1: flujo circular; MI2: flujo oxigenado; MI3: sin flujo). DC: diámetro corporal, DSV: diámetro del saco vitelino, A-natatoria: adquisición natatoria, $\mathrm{T}^{\circ}$ : temperatura

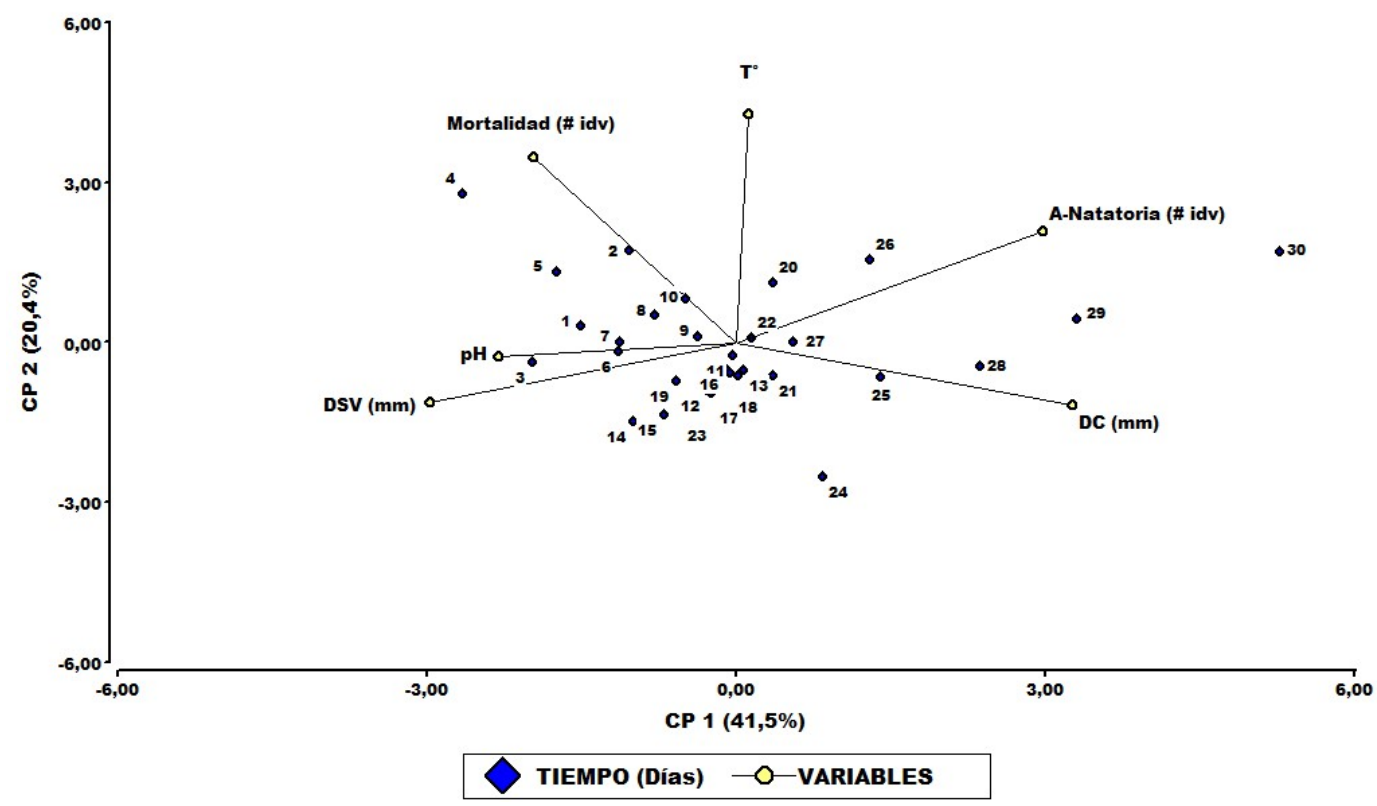

Figura 3. Análisis de componentes principales (ACP) con grafico biplot sobre el tiempo (días) de crecimiento de alevinos de Osteoglossum bicirrhosum del estadio larval dos al cinco, incubados en tres modelos de incubadora (MI1: flujo circular; MI2: flujo oxigenado; MI3: sin flujo) con respecto al tiempo (días). DC: diámetro corporal, DSV: diámetro del saco vitelino, A-natatoria: adquisición natatoria, $\mathrm{T}^{\circ}$ : temperatura 
variable diámetro del saco vitelino (DSV) se asocia más con los días 3 y 4 donde presentó los mayores valores, mientras que el $\mathrm{pH}$ presentó los valores más altos en los días 1 y 7 . Por otro lado, el segundo componente principal (CP2) con un aporte del $20.4 \%$ al extremo positivo de dicho componente se asocia con las variables mortalidad que presentó los valores más altos en los días 2,4 y 5 y en cuanto a la temperatura, los valores más altos se presentaron en los días 20 y 22.

\section{Literatura Citada}

1. Argumedo E, Rojas H. 2000. Manual de piscicultura con especies nativas. Colombia: Asociación de Acuicultores del Caqueta. $150 \mathrm{p}$.

2. Argumedo-Trilleras EG, Ramírez Chaux DA, Ramos Caldón HC, Franco Páez JE, Ochoa Endo SN, Ramírez Lugo CA, et al. 2017. Manual para la cría comercial de Arawana Plateada. Fortalecimiento-fomento y comercialización de la producción de larvas de arawana mediante la instalación de unidades rurales de cría en el Caquetá y Putumayo». $120 \mathrm{p}$.

3. Argumedo-Trilleras EG. 2009. Arawana azul manual para manejo de reproductores en cautiverio. Bogotá: Ed Produmedios. $96 \mathrm{p}$.

4. Argumedo-Trilleras EG, LópezMacias JN, Sánchez-Paéz C. 2007. Sistemas de producción de arawanas suramericanas, una alternativa de aprovechamiento sostenible de la biodiversidad íctica y de fortalecimiento de la acuicultura amazónica. Revista Electrónica de Ingeniería en Producción Acuícola 3: 99-100. Disponible en: http:/ /revistas.udenar.edu.co/index.php/reipa/ issue/view/194

5. Balbuena E, Ríos V, Flores A, Meza J. 2011. Manual para extensionista en acuicultura. Paraguay: Ministerio de Agricultura y Ganadería. 52 p. [Internet].
Disponible en: http://www.fao.org/3/aas828s.pdf

6. Bonilla-Castillo CA, Agudelo E. 2012. Indicaciones para la construcción de planes de manejo y conservación de arawana plateada (Osteoglossum bicirrhosum) en el río Putumayo, sector Puerto Leguízamo. Bogotá: Instituto Amazónico de Investigaciones Científicas SINCHI. $124 \mathrm{p}$.

7. Carillo-Estévez MA. 2009. Reproducción en peces aspectos básicos y sus aplicaciones. Madrid: Ed Paraninfo. 718 p.

8. Chaves-Moreno L, Chacón-Rodríguez L, Lozada-Morales J, Motta-Delgado P, Murcia-Ordoñez B. 2011. Evaluación de la reproducción inducida de cachama blanca (Piaractus brachypomus) con acetato de buserelina. Vet Zootec 6(1): 47-55.

9. Garzón-Ortíz WA, HawkinsCastañeda RS, Malagón-Ramírez JD. 2015. Elaboración del estudio de prefactibilidad para el montaje de una empresa de producción y exportación del pez arawana en Colombia. Tesis de Especialidad. Bogotá: Escuela Colombiana de Ingeniería Julio Garavito. $239 \mathrm{p}$.

10. Hernández-Olaya C. 2009. Estudio preliminar del levante de juveniles de arawana plateada (Osteoglossum bicirrhosum) en sistema cerrado de recirculación en la estación experimental Río Grande en el municipio de Cajica (Cundinamarca). Tesis de Bióloga. Bogotá: Univ Militar Nueva Granada. 77 p.

11. Hernández-Olaya C, Gómez-Ramírez E, Hurtado-Giraldo H. 2010. Estudio preliminar del levante de juveniles de arawana plateada (Osteoglosuum bicirrhosum) en sistemas cerrados de recirculación. Rev Fac Cienc Univ Militar Nueva 6: 96-113.

12. Landines-Parra MA, Sanabria-Ochoa AI, Victoria-Daza P. 2007. Producción de peces ornamentales en Colombia. Bogotá: Ed Universidad Nacional de Colombia. 240 p. 
13. Monaco G, Doroshov DI. 1983. Mechanical de-adhesion and incubation of white sturgeon eggs (Acipenser transmontanus Richardson) in jar incubators. Aquaculture 35: 117-123. doi: 10.1016/0044-8486(83)90079-0

14. Muñoz-Trujillo DM, Rojas AM, Murcia B, Chaves LC, Pimentel GA. 2017. Evaluación de dietas alternativas para alevines de arawanas (Osteoglossum bicirrhosum) en el Piedemonte Amazónico. Rev Vet 28: 145-151.

15. Piero-Ghezzi S, Requejo-Aleman JC, González-Guerreo S, Del Valle-Ayala O. 2015. Protocolo de reproducción arawana (Osteoglossum bicirrhosum). Lima: Fondo Nacional de Desarrollo Pesquero (FONDEPES). $11 \mathrm{p}$.

16. Prieto M, Olivera AM. 2002. Incubación artificial de huevos embrionados de tilapia roja Oreochromis sp. Rev Colomb Cienc Pec 15: 115-120.
17. Quintero-Pinto LG, Pardo-Gamboa BS, Qintero-Pardo AM. 2011. Manual técnico para la producción de peces de consumo a pequeña y mediana escala en el departamento de Cundinamarca. Secretaria de Agricultura y Desarrollo Rural de Cundinamarca. Bogotá: Universidad Nacional de Colombia. $92 \mathrm{p}$.

18. Ribeyro-Schult O, Ismiño-Orbe R, Chu-Koo F, Verdi-Olivares L, Verastegui-Tello M, Del Castillo JM. 2014. Crecimiento de alevinos de Osteoglossum Bicirrhosum «arahuana plateada» en ambientes controlados influenciados por frecuencias alimenticias. Rev Cienc Amazónica (Iquitos) 4: 45-53.

19. Salinas-Coy Y, Agudelo-Córdoba E. 2000. Peces de importancia económica en la cuenca amazónica colombiana. Bogotá: Instituto Amazónico de Investigaciones Científicas SINCHI. 140 p. 